\title{
Kikuchi Disease Causing Fever of Unknown Origin and Generalized Lymphadenopathy
}

\author{
Jumana ALBARAMKI, ${ }^{1}$ Rama JADALLAH, ${ }^{1}$ Marwan ADWAN, ${ }^{2}$ Tariq ALADILY ${ }^{3}$ \\ ${ }^{1}$ Department of Pediatrics, The University of Jordan, Amman, Jordan \\ ${ }^{2}$ Department of Medicine, Divison of Rheumatology, The University of Jordan, Amman, Jordan \\ ${ }^{3}$ Department of Hematopathology, The University of Jordan, Amman, Jordan
}

Kikuchi-Fujimoto disease (KFD) is a rare benign self-limiting disease with an unknown etiology that shares similar clinical and laboratory results with other diseases. It is reported in adults and children. ${ }^{1-3}$ In this article, we report a 13-year-old boy with prolonged fever, weight loss, hepatosplenomegaly, and generalized lymphadenopathy who was diagnosed to have KFD by lymph node biopsy.

A previously well 13-year-old boy was admitted with a four week history of high grade fever. He was initially treated as a case of recurrent tonsillitis and had received multiple courses of antibiotics without any improvement. Initially, the fever was intermittent, then became continuous in the last two weeks. There was a history of generalized arthralgia and weight loss. There was no history of travel abroad, exposure to pets, tuberculosis, insect bite or ingestion of raw milk. There was no history of skin rash, arthritis, diarrhea or abdominal pain. Examination showed only a small, soft, non-tender palpable submandibular lymph node. The abdomen was not distended. No organomegaly was detected.

His complete blood count revealed mild hypochromic microcytic anemia with leucopenia. His erythrocyte sedimentation rate and $\mathrm{C}$-reactive protein were elevated. The lactate dehydrogenase was raised to $1556 \mathrm{U} / \mathrm{L}$ (normal 240-480 U/L). Ferritin was elevated at 1177 ng/mL (normal 21.8-274.6 ng/mL). Blood and urine cultures grew no organism. Serological tests for cytomegalovirus, Epstein-Barr virus, Brucella, Rickettsia and Salmonella typhi were negative. Rheumatoid factor, anti-nuclear, antidouble-stranded deoxyribonucleic acid, anti-extra nuclear, and anti-neutrophil cytoplasmic antibodies were not detected. Cardiac echocardiography was normal. Complete ophthalmological examination showed no evidence of uveitis. An abdominal ultrasound showed hepatomegaly and mild splenomegaly. Contrast enhanced computed tomography showed bilateral mildly enlarged deep cervical lymph nodes, multiple mildly enlarged enhancing axillary lymph nodes, and multiple moderately enlarged mesenteric, common iliac, external iliac, and inguinal lymph nodes.

Bone marrow trephine biopsy showed slightly hypocellular marrow for the patient's age, with trilineage hematopoiesis. Cervical lymph node excision was performed, which showed large areas of geographic necrosis with prominent nuclear debris and sheets of plasmacytoid dendritic cells. The background consisted of histiocytes, lymphocytes, and few eosinophils. The residual lymph node showed reactive germinal centers and tingible body macrophages, but no hemophagocytosis (Figure 1). These findings were 


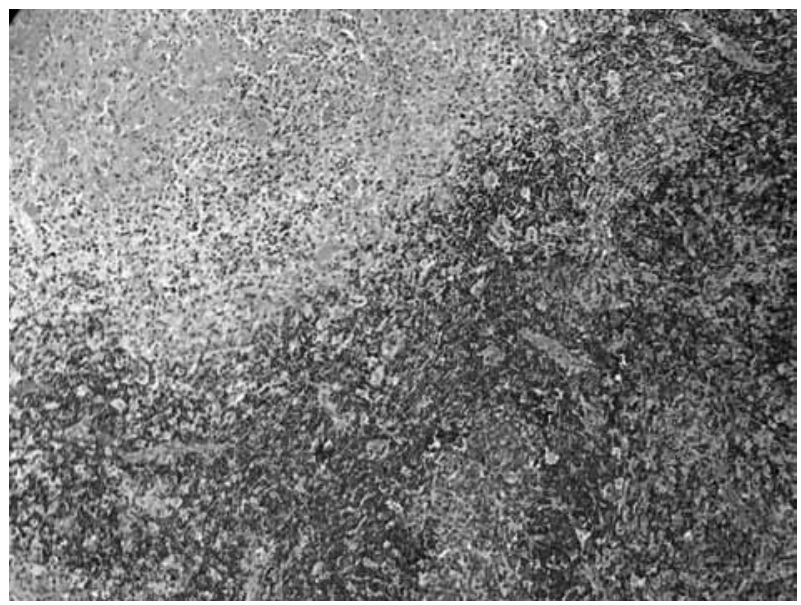

Figure 1. Lymph node excisional biopsy: wide area of geographic necrosis is present, while viable lymph node displays reactive germinal center and starry-sky appearance $(\mathrm{H}-\mathrm{E} \times 200)$.

consistent with necrotizing lymphadenitis of KFD. During the course of investigations, the high grade daily spiking fever persisted for twelve days and then resolved by itself.

Kikuchi-Fujimoto disease should be considered in every child with fever of unknown origin and lymphadenopathy if other infectious, autoimmune and malignant causes were excluded. It usually presents with fever and cervical lymphadenopathy in association with other systemic symptoms., ${ }^{4,5}$ Rarely, other lymph nodes such as inguinal and axillary lymph nodes may be involved. ${ }^{6,7}$ Laboratory examination usually shows anemia, leukopenia and elevated transaminases, lactate dehydrogenase and ferritin. ${ }^{5} \mathrm{~A}$ high index of suspicion is needed for its diagnosis by histological examination of the affected lymph nodes to establish an early diagnosis, and avoid other unnecessary investigations and treatments.

\section{Declaration of conflicting interests}

The authors declared no conflicts of interest with respect to the authorship and/or publication of this article.

\section{Funding}

The authors received no financial support for the research and/or authorship of this article.

\section{REFERENCES}

1. Kang HM, Kim JY, Choi EH, Lee HJ, Yun KW, Lee H. Clinical Characteristics of Severe Histiocytic Necrotizing Lymphadenitis (Kikuchi-Fujimoto Disease) in Children. J Pediatr 2016;171:208-12.

2. Lee KY, Yeon YH, Lee BC. Kikuchi-Fujimoto disease with prolonged fever in children. Pediatrics 2004;114:752-6.

3. Kim TY, Ha KS, Kim Y, Lee J, Lee K, Lee J. Characteristics of Kikuchi-Fujimoto disease in children compared with adults. Eur J Pediatr 2014;173:111-6.

4. Bosch X, Guilabert A, Miquel R, Campo E. Enigmatic Kikuchi-Fujimoto disease: a comprehensive review. Am J Clin Pathol 2004;122:141-52.

5. Kucukardali Y, Solmazgul E, Kunter E, Oncul O, Yildirim S, Kaplan M. Kikuchi-Fujimoto Disease: analysis of 244 cases. Clin Rheumatol 2007;26:50-4.

6. Pal Saini A, Jamil S, Dulai M. Kikuchi's disease causing persistent fever and iliac lymphadenopathy in an 8-year-old boy. Clin Pediatr (Phila) 2010;49:601-4.

7. de Gaudio M, Moshal K, Malone M, Novelli V. Kikuchi-Fujimoto disease causing fever of unknown origin in a nine-year-old boy. Scand $J$ Infect Dis 2010;42:946-9. 